\title{
Power Flow Analysis Of Nonlinear Finite Difference Model Of Bionic Acoustic Sensing Device
}

\author{
Qingsheng Wang ${ }^{a}$, Li Cuib and Xin Jiang ${ }^{\mathrm{c}}$ \\ Faculty of Engineering, Shanghai Polytechnic University, China \\ aqswang@sspu.edu.cn, bcuili@sspu.edu.cn, ${ }^{\mathrm{c}}$ jiangxin@sspu.edu.cn
}

\section{Keywords: Power Flow, Finite Difference Model, Acoustic Sensing Device}

\begin{abstract}
Parasitoid fly Ormia Ochracea has a remarkable ability to detect the direction of incident sound stimulus via its unique acoustic sensory organs. Based on the anatomy of them, a bionics acoustic sensing device is designed to mimic the localization function of the ears of the parasitoid fly Ormia ochracea. In this paper, the nonlinear dynamic model for the bionic acoustic sensing device is established. The dynamic characteristics of the nonlinear mechanical model and influence of the nonlinear factors under different exciting condition are estimated by means of finite difference method. These works will establish theoretical basis of the bionic acoustic sensor's design.
\end{abstract}

\section{Introduction}

Microphone arrays have been used to locate an aerial sound source for many years, these arrays usually consist of 2, 3 microphones or even more ${ }^{[1-3]}$. The arrival times of the sound stimulus applied to these microphones are determined and sent to the arithmetic-logic sections which could find out position of the sound sources later on.

The distance between each two microphones placed in the space is about $20 \mathrm{~cm}$, or equivalent amount, so that the arrival times of the sound pressures at these microphones can be detected and processed by the subsequent arithmetic-logic sections. The sizes of these appliances are always big relatively, and they usually consist of many components.

These physical characters of the structures mentioned above sometimes will narrow their applications in industry. And if the microphones, in other words, the acoustic sensory devices, can be placed in very close proximities to each other, these types of sound source localizers will have broader applications definitely.

An analysis is presented of mechanical responses to a sound field of the ears of the parasitoid fly Ormia ochracea [4-6]. It shows that the ears of this kind of parasitoid fly have a cuticular structure which can couple the ears mechanically in order to achieve the directional sensitivity. Based on the mechanism of the directional hearing of the parasitoid fly Ormia ochracea, some devices were invented as mimic products of the acoustic organ of the parasitoid fly Ormia ochracea [7].

In this paper, the mechanisms of the parasitoid fly Ormia ochracea for sound source localization are discussed, based upon this, a mini instrument for sound source localization is designed to accomplish the purpose of localizing the sound source by a relatively compact structure.

Analysis of the dynamic behavior of this instrument shows that incident angles of the sound have special relationship to the responses of this instrument, and the incident angles can be estimated by detecting the vibrations of it.

\section{Mechanisms of the Parasitoid Fly Ormia Ochracea for Sound Source Localization}

The structure of the ears of the parasitoid fly Ormia ochracea and its simplified mechanical modal is shown [4] in Figure.1 and Figure.2.

Let $\mathrm{x} 1$ and $\mathrm{x} 2$ denote the displacement of the two ends of the intertympanal bridge shown in Figure. $2, \mathrm{p}$ denote the incident pressure at the pivot point, $\mathrm{f} 1$ and $\mathrm{f} 2$ denote the ipsilateral and contralateral forces added up to the two sides of the system. 

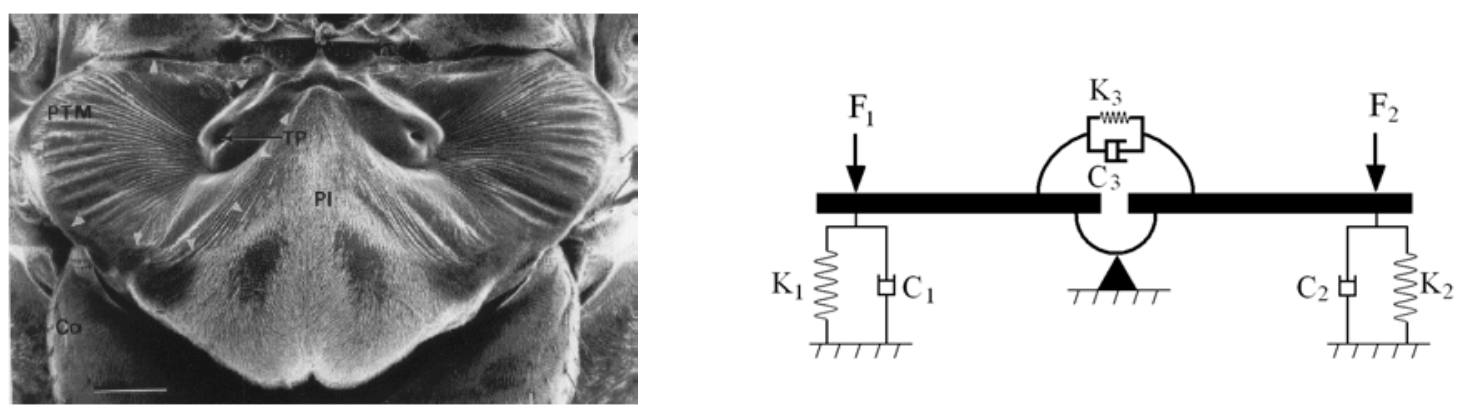

Figure 1. Hearing organ of Ormia ochracea Figure 2. Mechanical model of the hearing organ

The vibration equation of this mechanical modal can be expressed ${ }^{[4]}$ as:

$$
\left[\begin{array}{cc}
k_{1}+k_{3} & k_{3} \\
k_{3} & k_{2}+k_{3}
\end{array}\right] X+\left[\begin{array}{cc}
c_{1}+c_{3} & c_{3} \\
c_{3} & c_{2}+c_{3}
\end{array}\right] \dot{X}+\left[\begin{array}{cc}
m & 0 \\
0 & m
\end{array}\right] \ddot{X}=F
$$

The transfer functions between the displacement $x_{1}$ and $x_{2}$ and the incident pressure at the pivot point $p$ can be shown [4] as:

$$
\begin{gathered}
H_{x_{1} p}=\frac{s\left(k_{3}+i \omega c_{3}\right) \times\left(e^{j \omega \tau / 2}-e^{-i \omega \tau / 2}\right)+s\left(k+i \omega c-m \omega^{2}\right) e^{i \omega \tau / 2}}{\left(k+i \omega c+k_{3}+i \omega c_{3}-m \omega^{2}\right)^{2}-\left(k_{3}+i \omega c_{3}\right)^{2}} \\
H_{x_{1} p}=\frac{s\left(k_{3}+i \omega c_{3}\right) \times\left(e^{-i \omega \tau / 2}-e^{i \omega \tau / 2}\right)+s\left(k+i \omega c-m \omega^{2}\right) e^{-i \omega \tau / 2}}{\left(k+i \omega c+k_{3}+i \omega_{3}-m \omega^{2}\right)^{2}-\left(k_{3}+i \omega c_{3}\right)^{2}}
\end{gathered}
$$

From Eqs. (2) and (3), let $H_{x 1 x 2}$ denotes the proportion of $H_{x l p}$ and $H_{x 2 p}$, we may obtain Eq. (4):

$$
H_{x_{1} x_{2}}(\omega)=\frac{H_{x_{1} p}}{H_{x_{2} p}}=\frac{s\left(k_{3}+i \omega \omega_{3}\right) \times\left(e^{i \omega \tau / 2}-e^{-i \omega \tau / 2}\right)+s\left(k+i \omega c-m \omega^{2}\right) e^{i \omega \tau / 2}}{s\left(k_{3}+i \omega c_{3}\right) \times\left(e^{-i \omega \tau / 2}-e^{i \omega \tau / 2}\right)+s\left(k+i \omega c-m \omega^{2}\right) e^{-i \omega \tau / 2}}
$$

The calculated values of the incident angles $\theta$ can be derived from:

$$
\sin (\theta)=\frac{c \cdot \ln \left(\frac{\left(k_{3}+i \omega c_{3}+k+i \omega c-m \omega^{2}\right) H_{x_{1} x_{2}}+k_{3}+i \omega c_{3}}{k_{3}+i \omega c_{3}+k+i \omega c-m \omega^{2}+H_{x_{1} x_{2}}}\right)}{\omega d}
$$

\section{Nonlinear Model of Bionic Structure}

In order to localize the sound resource in three-dimensional space, we will introduce a mini instrument in this section. The structure of the auditory sensation devices in this instrument is shown in Figure.3. 


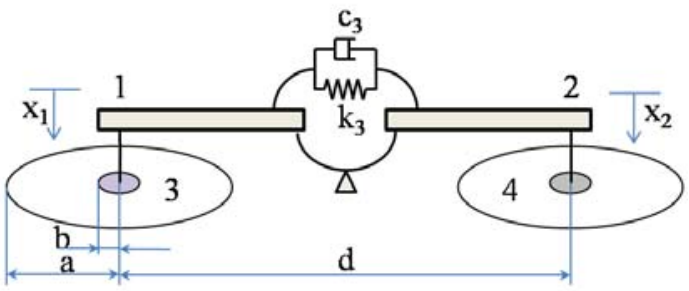

Figure 3. mechanical model bionic structure

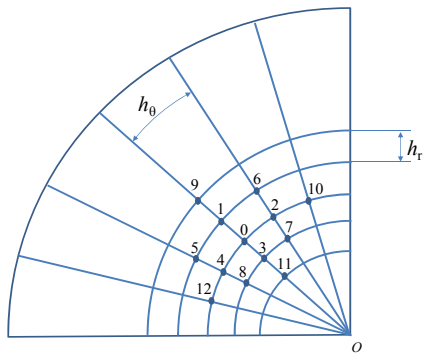

Figure 4. Discretization of the diaphragm

The coupling spring $\mathrm{k} 3$ is a nonlinear spring, the spring force can be given by:

$$
F\left(x_{1}+x_{2}\right)=k_{31}\left(x_{1}+x_{2}\right)+k_{32}\left(x_{1}+x_{2}\right)^{2}+k_{33}\left(x_{1}+x_{2}\right)^{3}
$$

As in Figure.3 and Figure.4, The vibration equation of the point 3, 4 in this mechanical modal can be expressed as:

$$
D \nabla^{4} W=q_{t}+q_{m}+q_{k}
$$

For the other points in the diaphragm, it can be given out as:

$$
D \nabla^{4} W=q_{t}+q_{m}
$$

Where $\mathrm{D}$ is the composite bending modulus, $\mathrm{W}$ the normal displacement, $q_{\mathrm{t}}$ the exciting load, $q_{\mathrm{m}}$ the inertia force, and $q_{\mathrm{k}}$ the spring force, can be derived as following.

$$
\begin{gathered}
D \nabla^{4} W=20 W_{0}-8\left(W_{1}+W_{2}+W_{3}+W_{4}\right)+2\left(W_{5}+W_{6}+W_{7}+W_{8}\right)+\left(W_{9}+W_{10}+W_{11}+W_{12}\right) \\
q_{t}=p \cdot \bar{s} \\
q_{m}=-\bar{m} \frac{d^{2} W}{d t^{2}} \\
q_{k}=F\left(x_{1}+x_{2}\right)
\end{gathered}
$$

As the phase locus and Poincaré section map when incident sound pressure 100pa shown in Figure.5, when the incentive intensity is relatively low, the nonlinear characteristics of this system are not very remarkable. But when the sound pressure increase to $1 \mathrm{Mpa}$, the nonlinear characteristics occur obviously, as shown in Figure.6.

\section{Power Flow Analysis of the Nonlinear Finite Difference Model}

Power always flow from ipsilateral side to contralateral side although the mechanical energy contained in ipsilateral side is higher than contralateral side as shown in Figure 7 , where $\mathrm{I}_{2}-\mathrm{I}_{1}$ is the power flow from ipsilateral side to contralateral side. 
When the stiffness of coupling spring $\mathrm{k} 3$ is very small, the power flows slightly from ipsilateral side to contralateral side. The both sides vibrate almost desperately in such a case. As $\mathrm{k} 3$ increases the power flow will increase simultaneously, the peak value of this curve indicates the maximum power flow efficiency.
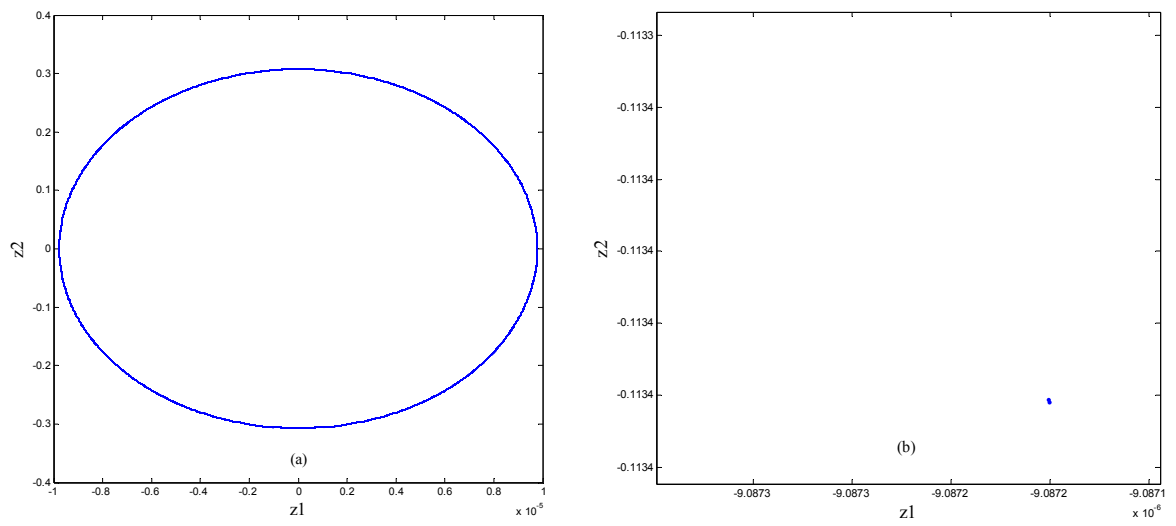

Figure 5. Phase locus and Poincaré section map when incident sound pressure 100pa
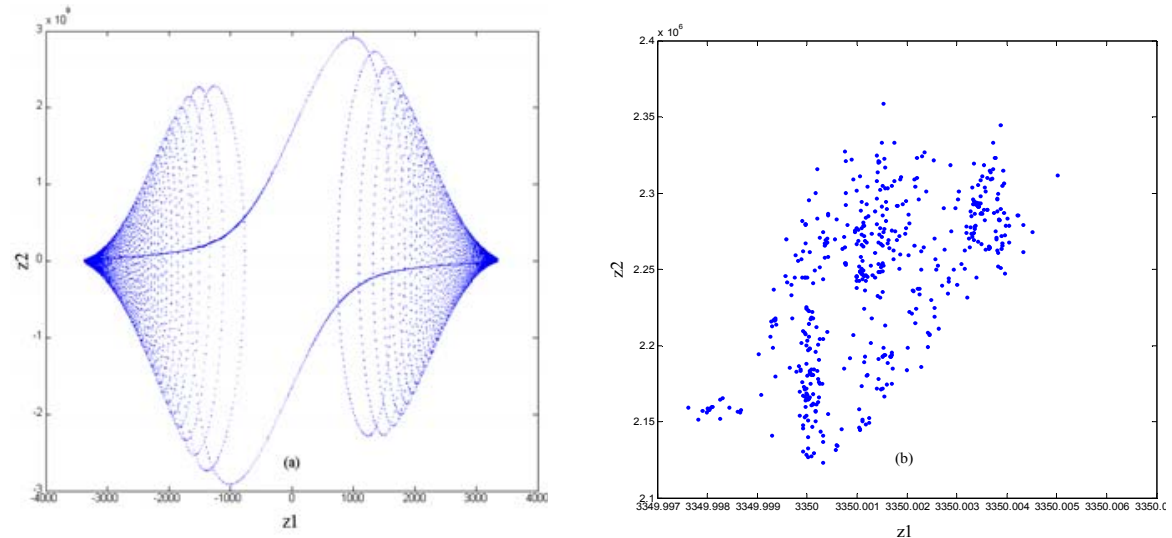

Figure 6. Phase locus and Poincaré section map when incident sound pressure 1Mpa

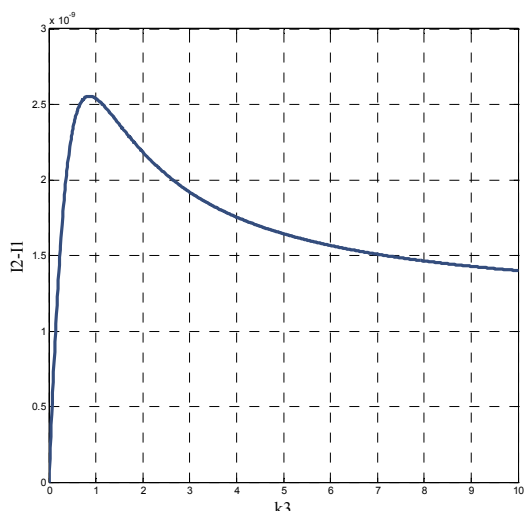

Figure 7. the power flow from ipsilateral side to contralateral side

\section{Conclusion}

In this paper, a nonlinear mechanical model for sound source localization is introduced, and its mechanism is discussed. The localization algorithm is given according to the analysis of the dynamic behaviour of this model. The dynamic characteristics of the nonlinear mechanical model and influence of the nonlinear factors under different exciting condition are estimated by means of finite difference method. Power flow analysis of the nonlinear finite difference model indicate that Power 
always flow from ipsilateral side to contralateral side although the mechanical energy contained in ipsilateral side is higher than contralateral side.These works will establish theoretical basis of the bionic acoustic sensor's design.

\section{Acknowledgment}

This work was financially supported by Young Teachers Training Project of Shanghai Polytechnic University (B50YC150005-4-11).

\section{References}

[1] Futoshi Asano, Hideki Asoh, Toshihiro Matsui, Sound Source Localization and Separation in Near Field, IEICE TRANSACTIONS on Fundamentals of Electronics, Communications and Computer Sciences. Vol.E83-A No.11, 2000, pp.2286-2294.

[2] Tsuji.T, Yamamoto.K, Ishii.I, Real-time Sound Source Localization Based on Audiovisual Frequency Integration. Pattern Recognition, ICPR 18th International Conference Volume 4, 2006, pp.:322 - 325 .

[3] Jie Huang, Ohnishi N, Sugie N., A Biomimetic System for Localization and Separation of Multiple Sound Sources. Instrumentation and Measurement," IEEE Transactions, Volume 44, Issue 3, 1995, pp.733- 738 .

[4] Miles, R.N, Robert, D, Hoy, R.R., Mechanically coupled ears for directional hearing in the parasitoid fly Ormia ochracea. Journal of the Acoustical Society of America, v 98, n 6, Dec, 1995, pp.3059-3070.

[5] D.Robert, R.N.Miles, R.R.Hoy, Tympanal mechanics in the parasitoid fly Ormia ochracea: intertympanal coupling during mechanical vibration. J comp Physiol A, 183, 1998, pp.443-452.

[6] Daniel Robert, Urban Willi, The histological architecture of the auditory organs in the parasitoid fly Ormia ochracea. Cell Tissue Res 301, 2000, pp.447-457.

[7] A Saito, N Ono, S Ando, Micro gimbal diaphragm for sound source localization with mimicking Ormia Ochracea. Proceedings of the 41st SICE Annual Conference, SCIE 2002, Aug.5-7, Osaka, pp.1640-1643. 